\title{
Research on IoT Malware Based on the ATT\&CK Model
}

\author{
Bo Hao, Fei Kang ${ }^{+}$, Ju Yang and Xiaobing Xiong \\ State Key Laboratory of Mathematical Engineering and Advanced Computing \\ Zhengzhou, Henan 450001, China
}

\begin{abstract}
The security of the IoT has become a hot research area in cyberspace security, among which the malware is a major threat. Based on the ATT\&CK model, this paper studies the composition and behavior of IoT malware, constructs a malicious behavior model of IoT malware, and analyzes the technical implementation of each tactic in the malicious behavior model of IoT malware from three aspects: operating system related, target environment related and specific tools related. Based on this, we finally propose the evolution direction of IoT malware, which will be conducive to a more comprehensive grasp of the characteristics of IoT malware, and be supportive for maintaining the security of IoT.
\end{abstract}

Keywords: IoT, ATT\&CK model, IoT malware, malicious behavior model.

\section{Introduction}

Nowadays, millions of embedded devices are omnipresent in daily life, such as the video surveillance systems, the industrial control systems, critical infrastructure [1], home automation [2], medicine [3] and smart cities [4], which bring great convenience to our lives. It is predicted that the number of IoT devices will increase from 15.4 billion in 2015 to 75.4 billion in 2025 [5]. But at the same time, due to the inherent defects of embedded devices [6], as well as the developers' insufficient considerations of security design, security incidents of IoT occur frequently. The typical case is that, on October 21, 2016, the huge botnet composed of Mirai set the largest Distributed Denial of Service (DDos) attack in history, reaching 1 Tbps [7].

In order to systematically and deeply analyse the composition, basic behavior and technical characteristics of IoT malware, this paper conducts research based on the ATT\&CK model.

Adversarial tactics, techniques, and common knowledge (ATT\&CK) is a model summarized by MITRE company, which describes the techniques used in each stage of attack from the perspective of attackers. The content of the model is extracted according to the data of real tracked network attacks. The framework of "Counter Tactics, Techniques and Common Sense" provided by it is a selected knowledge base composed of 12 tactics and more than 240 techniques that attackers will use when executing attacks, which is still being updated constantly [8].

The ATT\&CK model is divided into technical domain and platform domain as shown in Table 1:

Table 1: Division of ATT\&CK Model Domain

\begin{tabular}{|l|l|}
\hline Technology Domains & Platform(s) defined \\
\hline Enterprise & Linux, macOS, Windows, Cloud, Network \\
\hline Mobile & Android, iOS \\
\hline
\end{tabular}

In ATT\&CK model, "Tactics" is the tactical target of attackers' actions, which covers the standard and higher-level representation of what attackers do during operations. "Technique" refers to the way that attackers achieve tactical goals by executing actions. In the ATT\&CK model, we can see the relationship between tactics and techniques, and there may be many methods or techniques to achieve the same tactical goal.

At present, the ATT\&CK model mainly applies in the following scenarios :

\footnotetext{
+ Corresponding author.

E-mail address: 18638130587@163.com.
} 
Firstly, it is applied to simulate red and blue confrontation. The ATT\&CK model can be used to create confrontational simulation scenarios to test and verify defense strategies against common countermeasures.

Secondly, it is applied to assess the gap of defense. The ATT\&CK model can be used as a common confrontation model with behavior as the core to evaluate tools, monitoring and mitigation measures in existing defense strategies in organizations and enterprises.

Thirdly, it is applied to evaluate the maturity of Security Operations Centre (SOC). The ATT\&CK model can be used as a measure to determine the effectiveness of SOC in detecting, analyzing and responding to invasion.

Fourthly, it is applied to collect the network threat intelligence. The ATT\&CK model describes confrontation behavior in a standard way.It can track the attacker according to the techniques and tactics in ATT\&CK model.

Based on the ATT\&CK model, this paper constructs the malicious behavior model of IoT malware, presents the tactical objectives of each stage, and analyses the technical implementation from three aspects(including seven relevances) according to the necessary conditions of technique implementation in each tactical stage, and puts forward the evolution direction of IoT malware.

\section{Related Work}

According to the open source IoT malware and the samples captured by honeypot of IoT malware, researchers deeply focused on particular IoT malware, in the aspects of structure, behavior and how the IoT malware realize their malicious functions. Antonakakis et al. [9] analysed in-depth the Mirai botnet, researched how the Mirai botnet emerged, what classes of devices were affected and discussed Mirai's structure and propagation in detail. Artur Marzano et al. [11] compared Bashlite with Mirai and discussed their differences from the aspects of support infrastructures, available attacks, attack targets and how operators managed their botnets. Their research provides the evidence that malicious activity are becoming more sophisticated. Sam Edwards et al.[18] analysed the sample of Hajime captured in the wild from the aspects of reconnaissance and infection, downloader stub and DHT downloader in detail, and described the process of communicates over a distributed/decentralized overlay network to receive configuration and software updates.

At the same time, other researchers horizontally compared and analysed the characteristics of different IoT malicious. Benjamin vignau et al. [14] compared the characteristic features of 16 most widespread IoT malware programs of the last decade, and summarized 15 malicious features of the malware programs. M. Shobana et al. [12] explored various types of IoT malware evolved from 2013 to 2017, including 7 kinds of IoT botnets and 2 kinds of IoT worms, spotted the vulnerable points in the IoT environment, and summarized the comparison of characteristics of different IoT malware. Michele De Dono et al. [10] provided an up-todate picture of DDoS attacks in the specific subject of the IoT, and studied how these attacks worked. Based on the above research, this paper focuses on the technical realization of IoT malicious and the necessary conditions it relies on.

\section{Main Components and Behaviors of IoT Malware}

\subsection{Characteristics of IoT Devices}

Before analyzing the main components and behaviors of IoT malware, we should first understand the characteristics of IoT devices. Combined with the paper [16], the characteristics of IoT devices as summarized as follows:

(1) Limited flash memory space mainly used to store embedded operating system and firmware.

(2) Limited computing power and low power consumption level.

(3) It is mainly ARM / MIPS.

(4) Most of them are connected with each other, some access the Internet.

(5) Most have integrated Busybox tools. 


\subsection{Main Components of IoT Malware}

Based on the open source IoT malware such as Mirai and BASHLITE, according to the existing research[9][11][16], the composition of IoT malware are summarized (shown in Fig. 1).

The IoT malware mainly consists of the following five parts:

(1) Bots. Bots are composed of the IoT embedded devices which run malware, receive the attacker's instructions, implement malicious behaviors such as DDoS attacks, and are responsible for scanning vulnerable IoT devices.

(2) Command and control (CNC) server. Through the CNC server, attackers can send attack instructions to the Bots, and the Bots keep heartbeat connection with CNC server.

(3) Report server. Report server will receive vulnerable IoT device information scanned by Bots.

(4) Loader server. Loader server will $\log$ in vulnerable IoT devices to realize the download and execution of malware on infected devices.

(5) Distribution server. It is the location where the cross compiled malware adapted to different platforms stored to be downloaded by infected IoT devices.

\subsection{Main Behaviors of IoT Malware}

The main behaviors of IoT malware are as follows:

(1) Scan. IoT malware will continuously scan the Internet to find vulnerable IoT devices. It usually scans Telnet ports or other open services reachable over Internet, trying to log in with hard coded credentials.

(2) Report. After logging in the target device successfully, IoT malware will report the IP address along with relevant certificate information of the target device to the report server.

(3) Initial Access. The Loader server access the target device with the credentials stored in the Report server and detect the platform architecture, operating system and other basic information of the target device.

(4) Download and Execute. The target device downloads and executes the malware of the corresponding architecture. IoT malware escalates privilege by exploits in IoT devices, monopolizes the relevant port, eradicates other malicious programs, hides its own files, communicates with the CNC server regularly, and scans other vulnerable IoT devices at the same time.

(5) Attack. IoT malware launches DDoS attack to the specified target, participates the infected device in "mine pool" and implements other malicious behaviors.

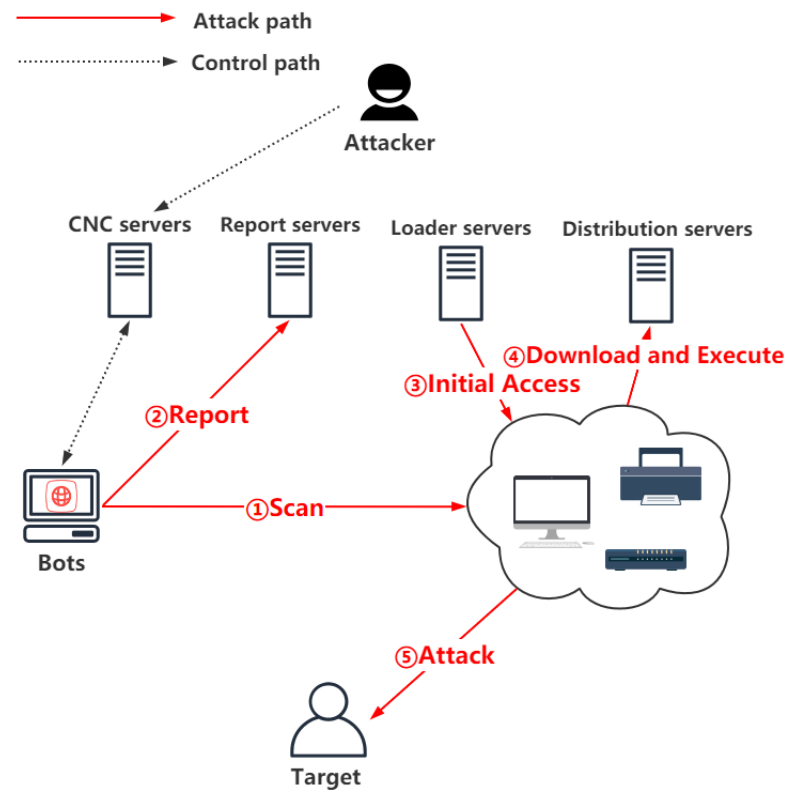

Fig. 1: The composition of IoT malware.

Based on the ATT\&CK model, compared with the behaviors of IoT malware, it is obvious that in the whole life cycle of IoT malware, it can map and reproduce the 12 tactics of the ATT\&CK model and the 
implementation of some techniques. By compacting the malicious behavior of IoT malware [25], this paper divides IoT malware's life cycle into the following seven tactical stages (shown in Table 2), and constructs a malicious behavior model of IoT malware (shown in Fig. 2).

Table 2: Seven tactical stages of IoT malware

\begin{tabular}{|c|c|c|c|c|c|c|c|}
\hline Tactical phase & Stage one & Stage two & Stage three & Stage four & Stage five & Stage six & Stage seven \\
\hline $\begin{array}{c}\text { ATT\&CK } \\
\text { Model }\end{array}$ & $\begin{array}{c}\text { Initial } \\
\text { Access }\end{array}$ & Execution & Persistence & $\begin{array}{c}\text { Privilege } \\
\text { Escalation }\end{array}$ & $\begin{array}{c}\text { Defense } \\
\text { Evasion }\end{array}$ & $\begin{array}{c}\text { Command } \\
\text { and control }\end{array}$ & Impact \\
\hline $\begin{array}{c}\text { IoT malware } \\
\text { Tactical Phase }\end{array}$ & Scan & $\begin{array}{c}\text { Dropper and } \\
\text { Exploit }\end{array}$ & Monopolize & $\begin{array}{c}\text { Privilege } \\
\text { Escalation }\end{array}$ & $\begin{array}{c}\text { Defense } \\
\text { Evasion }\end{array}$ & $\begin{array}{c}\text { Command } \\
\text { and Control }\end{array}$ & $\begin{array}{c}\text { Implement } \\
\text { Malicious } \\
\text { Behaviors }\end{array}$ \\
\hline
\end{tabular}

The main target of each tactical stage are described as follows:

(1) Scan. IoT malware initiates scanning to the public IP address to detect potential target devices.

(2) Dropper and Exploit. IoT malware finds the folder with writable and executable permissions in the target device, detects the target device architecture, downloads and executes the malware of the corresponding target architecture.

(3) Monopolize. IoT malware eradicates other processes, checks and deletes other suspicious malicious program files.

(4) Privilege Escalation. IoT malware obtains higher privilege through process injection, exploits, etc.

(5) Defense Evasion. IoT malware realizes anti debugging function, hides files and directories, prevents the target device from restarting, etc.

(6) Command and Control. IoT malware keeps heartbeat connection with CNC server and receives command from CNC server.

(7) Implement Malicious Behaviors. IoT malware accepts and analyzes attack command, launches DDoS attack; encrypts device data, participates in crypto-mining, etc.

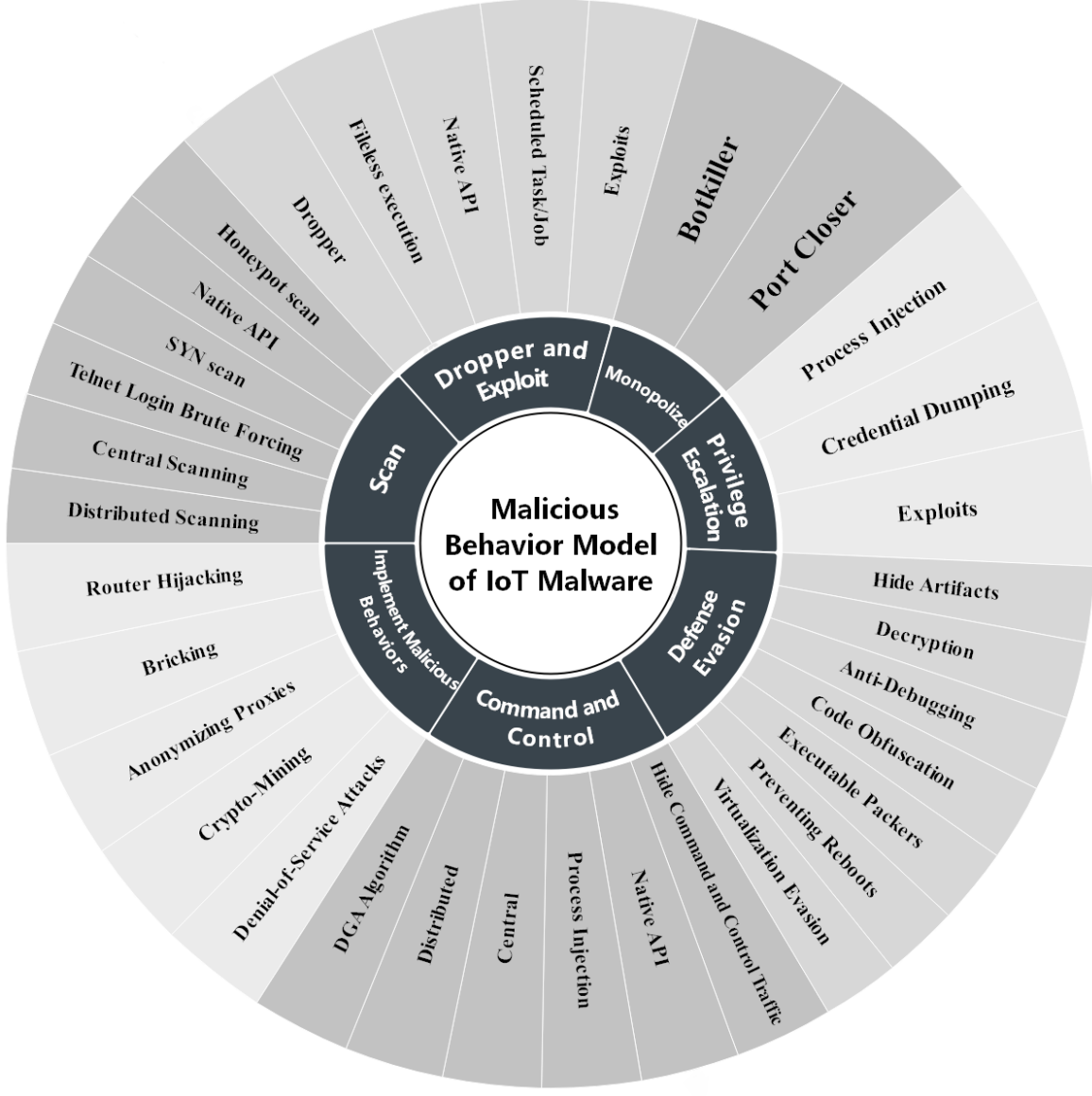

Fig. 2: The Malicious Behavior Model of IoT Malware. 


\section{Research on IoT Malware Technique Based on the ATT\&CK Model}

In the above seven tactical stages, each tactic has its corresponding technical support, and the realization of each technique has its necessary conditions and specific requirements. Some techniques need to invoke the specific system call. For example, IoT malware invokes system call memfd_create () to implement fileless execution. Some techniques are based on specific services or tools. For example, based on the basic commands provided by the Busybox tool, IoT malware completes the target device architecture detection. Some techniques need to consider the different circumstances of the target device. For example, IoT malware uses different exploits according to different target devices.

According to the necessary conditions needed in the implementations of technique, this chapter analyzes the techniques covered in the tactical stages of IoT malware from three aspects as follows.

\subsection{Related to Operating System}

Different operating systems provide different system services, follow different management mechanisms, and load different system kernels. The implementation of technique related to operating system refers to the specific conditions and constraints related to the operating system that the implementation of technique relies on, which are mainly divided into the following three types:

\section{A. Different System Calls and APIs}

Operating system kernel provides a series of multi-kernel functions with predetermined function, which are presented to users through a set of interfaces called system call. The system call transmits the request of the application program to the kernel, calls the corresponding kernel function to complete the required processing, and returns the processing result to the application program. Because different operating systems have different system kernels, their system calls are also different.

In the "Fileless execution" technique of the "Dropper and Exploit" tactic, IoT malware calls memfd_create(), which is a underlying system call to create a memory image file, which will not be mapped into the actual file system. In this way IoT malware realizes the startup of process without file, achieving the purpose of secretly executing the malicious programs.

The ptrace() system call enables a debugging process to observe and control another process (and each individual thread), including changing memory and register values, and is mainly used for debugging and tracking system calls. In the "Anti-Debugging" technique of "Defense Evasion" tactic, the IoT malware judges whether it is in the debugging environment by judging the return value based on the properties that ptrace() can only be invoked once on a process. When invoked multiple times on the same process, the call to ptrace() will fail and return -1. On the other hand, IoT malware preempts the process by invoking ptrace (PTRACE_TRACEME,pid,...), so that this process can no longer be traced by debugging.

A collection of all system calls provided by the operating system is called application programming interface (API). Native API provides users with a way to invoke the underlying operating system services in the kernel, such as file system, external devices, error handling mechanism, processes, threads and other services [15]. IoT malware can directly interact with API to perform various operations and behaviors.

In the "Native API" technique of "Command and Control" tactic, IoT malware realizes its communication, downloading, scanning and command transmission functions through socket API.

In the "Native API" technique of "Scan" tactic, IoT malware realizes TCP SYN scanning by constructing raw socket provided by API, which greatly improves the scanning efficiency.

\section{B. Different Operating System Management Mechanisms}

Operating system mainly implements the following three management mechanisms:

Firstly, process management mechanism, including process control, process synchronization, process communication and process scheduling. Secondly, memory management mechanism, including memory allocation, memory protection, address mapping and memory expansion. Thirdly, file management mechanism, including file storage space management, directory management, read/write management, file sharing and protection.

We analyse the technical realization of IoT malware from three relevances as follows: 


\section{(1) Process Management}

In the "Native API" technique of "Command and Control" tactic, when facing embedded systems using Linux kernel, IoT malware could adopt the following two ways to realize process startup and instruction execution. Firstly, malware could use glibc APIs. Specifically, they can invoke system(), fork (), or exec series functions, including execl (), execlp (), execle (), etc. Secondly, IoT malware executes instructions through syscall. By triggering "int 80 interrupt" directly through assembly, IoT malware directly uses the system call function provided by the operating system.

The "Process Injection" in the tactic of "Privilege Elevation" is a method of executing arbitrary code in the address space of a separate live process. Running code in the context of another process may allow access to the process's memory and system/network resources, so as to achieve the purpose of privilege elevation.

For the embedded Linux system, IoT malware has the following three process injection methods: Ptrace System Calls. IoT malware may inject malicious code into processes via ptrace() system calls, write arbitrary code into a running process, then invoke that memory with PTRACE_SETREGS to set the register containing the next instruction to execute. Proc Memory. Proc memory injection is commonly performed by overwriting the target processes' stack using memory mappings provided by the /proc filesystem(/proc/[pid]). VDSO Hijacking. IoT malware may hijack the syscall interface code stubs mapped into a process from the linux-vdso.so shared object to execute syscalls to open and map a malicious shared object.

\section{(2) Memory Management}

In the "Credential Dumping" technique of "Privilege Escalation" tactic, IoT malware generally obtains the real-time memory of running process through Proc filesystem or /proc, which may contain password information stored in the form of a hash or a clear text password, or dump the contents of /etc/passwd and letc/shadow to enable offline password cracking.

In the "Virtualization Evasion" technique of "Defense Evasion" tactic, IoT malware reads the files /sys/class/dmi/id/product_name and /sys/class/dmi/id/sys_vendor and compares the file contents with the keyword "VirtualBox" to check whether the device is a honeypot running in a virtual machine, which is adopted by Amnesia [26].

(3) File Management

In the "Hide Artifacts" technique of "Defense Evasion" tactic, IoT malware evades security detection by hiding files, directories, etc. In terms of hiding process names, IoT malware invokes $\operatorname{prctl}()$ or modify the argv [0] of process to hide their own processes. Mirai calls the $\operatorname{prctl}()$ system call with the PR_SET_NAME argument to make the process name into random string [21], which can not only evade detection, but also avoid being cleared by other IoT malwares. In terms of hiding file names, besides putting a "." as the first character in the file or folder name, the system call unlink() does not remove the data blocks from the file system, it simply removes the entry (filename) from the directory table and the file's data blocks remain reserved until all processes that have the file referenced close it or terminate. Mirai and Hajime adopt this method [25].

In the "Fileless execution" technique of "Dropper and Exploit" tactic, IoT malware uses the shared memory partition in the Linux file system to store process files, such as $/ \mathrm{dev} / \mathrm{shm}$ and $/ \mathrm{run} / \mathrm{shm}$. These directories are actually mounted on the allocated memory space of the file system, and the files written in these directories will not actually fall on the physical disk, so that the purpose of hiding itself can be achieved.

\section{Different Operating System Services}

System services refer to programs or processes that perform specified system functions, and usually run in the background. System services are favored by attackers, because they are usually related to managing the memory, controlling input and output devices, and managing network and file resources. 
In the "Scheduled Task/Job" technique of "Dropper and Exploit" tactic, IoT malware abuses task scheduling functionality, such as At, Cron and Systemed Timers, to facilitate initial or recurring execution of malicious code.

In the "Telnet Login Brute Forcing" technique of "Scan" tactic, IoT malware uses Telnet service to realize initial access to devices on the Internet through brute forcing.

\subsection{Related to Target Environment}

IoT malware will adopt the corresponding technique according to the characteristics of the target environment, such as the unique applications, specific mechanisms, etc.

The embedded devices usually implement watchdog, to prevent embedded system hanging for a long time without human intervention, which is essentially a counter. The hardware timer of watchdog is a timer that has a timeout set and which is reset by the daemon component at a specified interval. If the system is running so poorly that it is unable to send a timeout reset to the kernel driver module, a forced reset will occur on the system [19].

In the "Preventing Reboots" technique of "Defense Evasion" tactic, in order to prevent losing target device due to rebooting, IoT malware usually reset the counter, known as "feeding the dog", or disables the watchdog function by invoking ioctl() to send a control code.

In the "Exploits" technique of "Dropper and Exploit" and "Privilege Elevation" tactic, IoT malware may exploit software and protocols vulnerabilities in target devices to elevate privileges and execute malicious code.

For example, the early Hydra-2008.1 exploited authentication bypass vulnerability in D-Link routers [20]. The Darlloz worm exploits the "php-cgi" information leakage vulnerability in PHP to infect cameras, routers and other devices [21]. The new Mirai variant uses a code execution vulnerability in TR064/TR069 which can hijack or crash the device [21]. The Bashlite worm abuses the Shell-Shock vulnerability, which is a serious security vulnerability of Unix Bash Shell, to infect over one million Busybox devices [22].The IoT Reaper integrates a series of nine HTTP-based exploits, which all based on previously disclosed IoT vulnerabilities [25]. According to statistics, the number of CVE vulnerabilities involved in Echobot is as high as 73 [23].

In the "Router Hijacking" technique of "Implement Malicious Behaviors" tactic, through disclosed exploits, IoT malware modifies the DNS server settings and redirects all their DNS requests through a malicious DNS server, just as Tsunami [16].

\subsection{Related to Specific Tools}

In the malicious behavior model of IoT malware, the implementation of some techniques relies on a specific tool, program or protocol.

In the "Native API" technique of "Scan" tactic, IoT malware uses existing IoT devices search tools, such as Shodan, ZoomEye, etc. By combining the search engine API provided by them with the IoT malware's exploit module, it improved scanning and intrusion efficiency [17].

In the "Dropper" technique of "Dropper and Exploit" tactic, after logging into the target device through Telnet or SSH, IoT malware uses Dropper to download and execute binary files. Dropper can download and execute malicious binary files by means of Busybox basic tools integrated on the target devices. For example, IoT malware finds a writable directory through cat /proc/mounts, judges whether the command is normally executed through the error command echo information of /bin/busybox ECCHI, detects the target device's architecture by parsing echo ELF header, and downloads executable files through wget/ tftp /curl. If there is no download tool, IoT malware will transmit a simple downloader binary file written by the attacker through echo.

UPX is a compression tool for ELF binaries, which can achieve a compression ratio of $50 \%-70 \%$. Although the original purpose of the UPX is to reduce the size of the executable file, it has gradually be applied to anti-reversing and confusing code, which makes the static analysis of binary files more difficult. 
In the "Executable Packers" technique of "Defense Evasion" tactic, the Hajime worm uses a customized UPX tool to protect malicious programs by modifying the UPX header magic number [18].

In the "DGA Algorithm" technique of "Command and Control" tactic, IoT malware generates hundreds of pseudo-random domain names through DGA algorithm, and only selects one or several of them as real CNC server domain names to prevent being traced back [9].

In the "Anonymizing Proxies" technique of "Implement Malicious Behaviors" tactic, IoT malware integrates open source proxy tools into programs, providing traffic proxies for APT organizations or other network attacks to hide the original attack path of attackers [13].In the "Crypto-Mining" technique, the IoT malware adds the infected device to the "mine pool" by loading crypto-mining scripts or programs in the target device. This technique was first discovered in Linux.Darlloz in 2014 [14].

In the "Distributed" technique of "Command and Control" tactic, the Hajime worm uses BitTorrent's DHT protocol for peer discovery and uTorrent transport protocol for data exchange, to build a decentralized network structure [18].

To sum up, we briefly present the relevant specific conditions required in the implementation of techniques in each tactic from seven relevances (shown in Fig. 3).

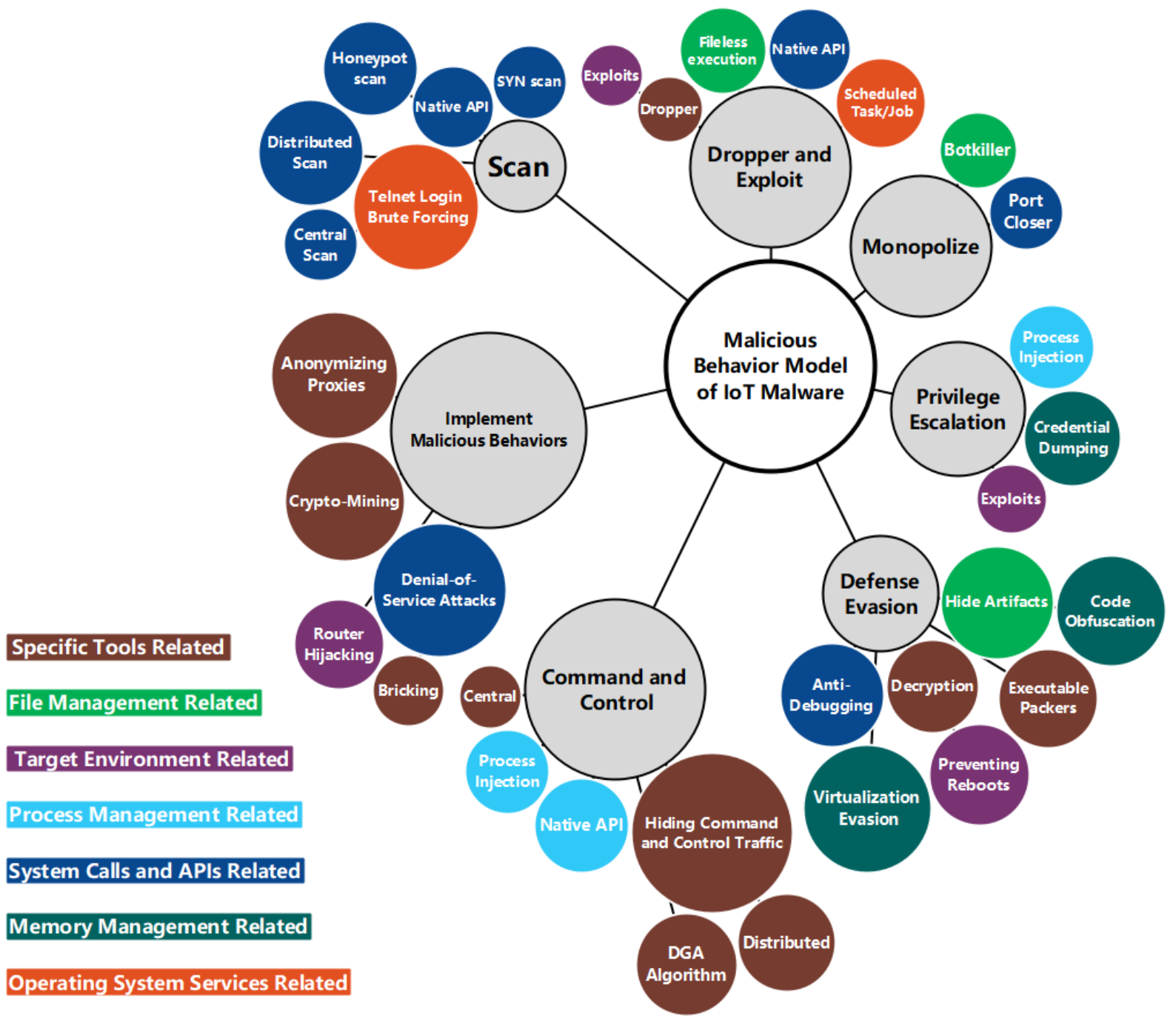

Fig. 3: The relevance of IoT malware technique implementation.

\section{Evolution Direction of IoT Malware}

The ultimate task of IoT malware is to be able to discover, infect, invade and implant target devices automatically and hiddenly, to run permanently, to control a large number of zombies stably, and to establish a large-scale botnet. In this process, it is bound to face the diversified devices in the IoT, resulting in the constraints related to the operating system, the target environment and the specific tools, in the 
implementation of IoT malware techniques. Faced with a large number of IoT devices with various types, diverse architecture, different operating systems and different in equipment resources, how to solve the universality of IoT malware technique has become a key issue in its development.

In this regard, we predict the evolution direction following of the IoT malware:

\subsection{Extensive Adaptability of Platform Architecture and Operating System}

There are various types of IoT device platforms, including ARM, MIPS, PowerPC, SPARC, etc. At the same time, the embedded operating system is various due to its different operating mechanisms, the tailorability of the kernel, the optional kernel modules, and the use of different lightweight support libraries, etc.

In the face of IoT devices with different platform architectures and operating systems, IoT malware must possess extensive adaptability, which could shield the limitations caused by the differences in underlying frameworks, and could be compatible with different platform architectures and operating systems, so that the implementations of tactical goals at various stages are not subject to the relevance of various platform architectures and operating systems, so as to ensure the normal operation of various functions in IoT malware.

\subsection{Adaptive Tailoring of Function Modules}

IoT devices play different roles and complete different tasks, resulting in huge differences in the size of the storage, computing, network and other resources. The IoT malware must realize the collection and judgment of the target device information and resources, and then flexibly tailors its own functional modules, comprehensively study and judge the target device resources and environmental conditions, so as to realize the variable form and function. IoT malware could implement the basic functions on target devices with limited resources such as computing and storage, and could implement the advanced malicious functions on target devices with abundant resources, so as to achieve target personalization of malicious function and maximize the utilization of target device resources.

\subsection{Diverse Malicious Behavior}

The NSFOCUS [24] pointed out that as of November 2020, the number of IoT-related vulnerabilities published by NVD platform has reached 1,541. The related vulnerabilities have the characteristics of low attack complexity and high hazard ratings. IoT vulnerabilities exploitation is a low-cost and high-yield attack method, and attackers are very concerned about the exploitation of IoT vulnerabilities, and follow up with some vulnerabilities very quickly. In the future, there will be more extensive vulnerabilities exploitation in IoT malware. At the same time, the ultimate goal of the IoT malware will not only be limited to launching DDos attacks, but will gradually develop into a variety of malicious behaviors such as data stealing, crypto mining, traffic hijacking, and ransomware attacking, etc.

\section{References}

[1] N. Cam-Winget, A.-R. Sadeghi, and Y. Jin, "Can IoT be secured:Emerging challenges in connecting the unconnected," in Proc.53rd ACM/EDAC/IEEE Design Automation Conference (DAC),2016, pp. 1-6.

[2] B. L. R. Stojkoska and K. V. Trivodaliev, "A review of Internet of Things for smart home: Challenges and solutions," Journal of Cleaner Production, vol. 140, pp. 1454-1464, 2017.

[3] B. Farahani, F. Firouzi, V. Chang, M. Badaroglu, N. Constant, and K. Mankodiya, "Towards fog-driven IoT ehealth: Promises and challenges of IoT in medicine and healthcare," Future Generation Computer Systems,vol. 78, pp. $659-676,2018$.

[4] A. Zanella, N. Bui, A. Castellani, L. Vangelista, and M. Zorzi, "Internet of things for smart cities," IEEE Internet of Things Journal, vol. 1,pp. 22-32, Feb 2014.

[5] Asosheh, Abbass \& Ivaki, Naghmeh. (2008). A comprehensive taxonomy of DDOS attacks and defense mechanism applying in a smart classification. WSEAS Transactions on Computers. 7. 281-290.

[6] Jing, Qi \& Vasilakos, Athanasios \& Wan, Jiafu \& Lu, Jingwei \& Qiu, Dechao. (2014). Security of the Internet of Things: Perspectives and challenges. Wireless Networks. 20. 2481-2501. 10.1007/s11276-014-0761-7. 
[7] H. Sinanović and S. Mrdovic, "Analysis of Mirai malicious software," 2017 25th International Conference on Software, Telecommunications and Computer Networks (SoftCOM), Split, Croatia, 2017, pp. 1-5, doi: 10.23919/SOFTCOM.2017.8115504.

[8] Strom B E, Applebaum A, Miller D P, et al. Mitre att\&ck: Design and philosophy[J]. Technical report, 2018.

[9] M. Antonakakis, T. April, M. Bailey, E. Bursztein, J. Cochran, Z. Du-rumeric, J. A. Halderman, D. Menscher, C. Seaman, N. Sullivan et al.,"Understanding the Mirai Botnet," in USENIX Security Symposium.USENIX, 2017.[J].

[10] Donno Michele-de, Draconi Nicola, Giaretta Alberto, et al. Analysis of DDoS-Capable IOT Maldives [C]//2017 Federated Conference on Computer Science and Information Systems (FEDCSIS): IEEE: 807-816.

[11] Marzano, Artur \& Alexander, David \& Fonseca, Osvaldo \& Fazzion, Elverton \& Hoepers, Cristine \& StedingJessen, Klaus \& Chaves, Marcio \& Cunha, Italo \& Guedes, Dorgival \& Meira Jr, Wagner. (2018). The Evolution of Bashlite and Mirai IoT Botnets. 00813-00818. 10.1109/ISCC.2018.8538636.

[12] Shobana M.,Dr.s.rathi. IOT Malware-An Analysis of IOT Device Hijacking[J]. International Journal of Scientific Research in Computer Science, Engineering and Information Technology.

[13] Geenens P. IoT botnet traits and techniques[J]. Botnets: Architectures, Countermeasures, and Challenges, 2019: 101.

[14] Vignau, Benjamin \& Khoury, Raphaël \& Hallé, Sylvain. (2019). 10 Years of IoT Malware: a Feature-Based Taxonomy. 10.1109/QRS-C.2019.0008.

[15] Chao Wang, Malicious Code Analysis Technology Based on Windows System Call Flowchart [D]. Beijing University of Posts and Telecommunications, 2015.

[16] Angrishi K. Turning Internet of Things(IoT) into Internet of Vulnerabilities (IoV) : IoT Botnets. ArXiv170203681 Cs. Published online February 13, 2017. Accessed March 6, 2021. http://arxiv.org/abs/1702.03681

[17] Al-Alami H, Hadi A, Al-Bahadili H. Vulnerability scanning of IoT devices in Jordan using Shodan[C]//2017 2nd International Conference on the Applications of Information Technology in Developing Renewable Energy Processes \& Systems (IT-DREPS). IEEE, 2017: 1-6.

[18] I. P. Sam Edwards, "Hajime: Analysis of a decentralized internet worm for iot devices," 2016.

[19] J. Margolis, T. T. Oh, S. Jadhav, Y. H. Kim and J. N. Kim, "An In-Depth Analysis of the Mirai Botnet," 2017 International Conference on Software Security and Assurance (ICSSA), Altoona, PA, USA, 2017, pp. 6-12, doi: 10.1109/ICSSA.2017.12.

[20] Costin A, Zaddach J. IoT malware: comprehensive survey, analysis framework and case studies[J]. BlackHat USA, 2018.

[21] Wang A, Liang R, Liu X, et al. An inside look at IoT malware[C]//International Conference on Industrial IoT Technologies and Applications. Springer, Cham, 2017: 176-186.

[22] Sebastian, Walla \& Rossow, Christian. (2019). MALPITY: Automatic Identification and Exploitation of Tarpit Vulnerabilities in Malware. 590-605. 10.1109/EuroSP.2019.00049.

[23] Khoury, Raphaël \& Vignau, Benjamin \& Hallé, Sylvain \& Hamou-Lhadj, Abdelwahab \& Razgallah, Asma. (2021). An Analysis of the Use of CVEs by IoT Malware. 10.1007/978-3-030-70881-8_4.

[24] NSFOCUS, National Engineering Laboratory of Network Security Emergency Technology, 2020 Annual Report on Internet of Things Security, 2020.[J]

[25] Georgios Kambourakis,Marios Anagnostopoulos,Weizhi Meng,Peng Zhou, Botnets: Architectures, Countermeasures, and Challenges[M]. CRC Press, 2019.

[26] Sørensen D A, Vanggaard N, Pedersen J M. Automatic profile-based firewall for iot devices[J]. 2017. 